\title{
Optimal Beamforming for Single Group Multicast Systems Based on Weighted Sum Rate
}

\author{
Bo Du, Ming Chen, Wence Zhang, and Cunhua Pan \\ National Mobile Communications Research Laboratory \\ Southeast University, Nanjing 210096, China \\ Email: \{dubo, chenming, wencezhang, cunhuapan\}@seu.edu.cn
}

\begin{abstract}
In this paper, a novel optimization objective namely weighted sum rate is proposed for beamformer design in single group multicast systems. As an upper bound and special case of maximizing minimum rate, it can overcome previous disadvantages of computation complexity and sensitivity to channel state when the weights are chosen properly. Although the corresponding optimization problem is nonconvex, some properties of the optimal solution and closed-form solutions under some usual special cases are derived. In addition, an iterative algorithm with low computation complexity for general case is proposed. Simulation results show that the beamformer obtained by the iterative algorithm not only improves the minimum rate and average rate, but also gets better fairness and overall performance compared with previous schemes.
\end{abstract}

Index Terms-single group multicast, beamforming, weighted sum rate.

\section{INTRODUCTION}

Modern mobile communication systems, such as LTE, LTEA and WiMAX, will support multicast. Recently there appear some researches on beamformer design for multi-antenna multicast systems [1]-[5], since multi-antenna transmission can improve the spectral efficiency greatly.

Different data is transmitted for individual user in multiuser unicast systems, so beamformers are designed respectively to improve each user's receiving performance and eliminate the inference among users. While same data is transmitted in single group multicast systems, all the users will share a common beamformer, which should guarantee all the users' receiving performance, particularly the users with poor channels, thus the design objective must be fair for all the users.

By now, maximizing minimum rate is the main concern for beamformer design in a single-group multicast system [2]-[4]. While maximizing minimum rate intends to provide Quality of Service (QoS) assurance for each user, so the overall performance will be limited by the worst user. However, in some cases, a poor user may reduce overall performance tremendously, especially when the channel of the worst user is uncorrelated with others, leading to that the system works at an extremely low rate. In other words, it may reduce the performance of those users who have good channels and deserve better service. But the poor users should not be neglected simply, hence all the users should be considered in different degrees. Meanwhile, since maximizing minimum rate is a NP-hard problem [2], it is difficult to obtain the optimal solution with acceptable complexity, except for some special case [4] or upper bound after relaxation [5]. Otherwise, only suboptimal solution could be obtained through semidefinite relaxation (SDR) [2] or successive orthogonalization [3].

In this paper, a new optimization objective namely maximizing weighted sum rate is proposed. It provides different priority for each user according to the channel quality. Compared with maximizing minimum rate, maximizing weighted sum rate shifts the focus from the worst user to the worse user group. In addition, maximizing minimum rate can be regarded as a lower bound and a special case of maximizing weighted sum rate. This objective can guarantee minimum rate, average rate and fairness when the weights in the objective are chosen properly. In this paper, the weights are chosen inversely proportional to the channel quality such that the worse the channel quality is, the higher the weight is.

The obtained original optimization problem is nonconvex, some properties of the optimal solution and closed-form solutions under some usual special cases are derived. Furthermore, a low-complexity iterative algorithm for general case is proposed, whose initial value is the solution of maximizing weighted SNR.

Notation: Uppercase and lowercase boldface letters denote matrices and vectors respectively, $\mathbf{A}^{\mathrm{T}}$ and $\mathbf{A}^{\mathrm{H}}$ denote the transpose and conjugate transpose of $\mathbf{A},\|\mathbf{x}\|$ denotes the Euclidean norm of $\mathbf{x}, E\{\cdot\}$ is the expectation of a random variable, $\mathbf{0}_{M}, \mathbf{1}_{M}$ and $\mathbf{I}_{M}$ denote the $M \times 1$ all zeros vector, $M \times 1$ all ones vector and $M \times M$ identity matrix, respectively.

\section{Problem Statement}

Consider a base station with $M$ antennas to transmit common data to $N$ single-antenna users. Transmitted symbol is $s \in \mathbb{C}$, subjected to $E\left\{|s|^{2}\right\}=P$, where $P$ is the maximum transmit power. Denote $\mathbf{w} \in \mathbb{C}^{M \times 1}$ the beamformer applied to the $M$ transmit antennas, which fulfills the transmit power constraint $\|\mathbf{w}\|^{2} \leq 1$.

All the channels are assumed to be i.i.d. Rayleigh, so the signal received by user $i$ can be expressed as

$$
y_{i}=\mathbf{h}_{i}^{\mathrm{H}} \mathbf{w} s+z_{i}
$$

where $\mathbf{h}_{i} \in \mathbb{C}^{M \times 1}$ is channel vector of user $i$ distributed as $\mathcal{C N}\left(0, \mathbf{I}_{M}\right)$ that is constant for several frames before changing, $z_{i}$ is additive noise of user $i$ distributed as $\mathcal{C N}\left(0, \sigma_{i}^{2}\right)$. The transmitter is assumed to accurately know the instantaneous channel state information of all users. 
The instantaneous rate of user $i$ is

$$
R_{i}=\log _{2}\left(1+\frac{P\left|\mathbf{h}_{i}^{\mathrm{H}} \mathbf{w}\right|^{2}}{\sigma_{i}^{2}}\right)=\log _{2}\left(1+\mathbf{w}^{\mathrm{H}} \mathbf{Q}_{i} \mathbf{w}\right)
$$

where $\mathbf{Q}_{i}=\left(P \mathbf{h}_{i} \mathbf{h}_{i}^{\mathrm{H}}\right) / \sigma_{i}^{2}$.

When maximizing weighted sum rate is chosen as the optimization objective, the problem can be modeled as

$$
\begin{cases}\min _{\mathbf{w}} & f(\mathbf{w})=-\sum_{i=1}^{N} \alpha_{i} \log _{2}\left(1+\mathbf{w}^{\mathrm{H}} \mathbf{Q}_{i} \mathbf{w}\right) \\ \text { s.t. } & \|\mathbf{w}\|^{2} \leq 1\end{cases}
$$

where $\alpha_{i}$ is the weight of user $i$ satisfying $\sum_{i=1}^{N} \alpha_{i}=1$. In this paper and simulation, we choose

$$
\alpha_{i}=\frac{\sigma_{i}^{2} /\left\|\mathbf{h}_{i}\right\|^{2}}{\sum_{j=1}^{N}\left(\sigma_{j}^{2} /\left\|\mathbf{h}_{j}\right\|^{2}\right)}
$$

which is determined by the channel state, the worse the channel is, the higher the corresponding weight is.

Remark 1: Because $\min _{i=1, \cdots, N} R_{i} \leq \sum_{i=1}^{N} \alpha_{i} R_{i}$, weighted sum rate can be regarded as an upper bound of the minimum rate, i.e., the objective in [2]. Furthermore, minimum rate is a special case of weighted sum rate with weight $(1,0, \cdots, 0)$.

Remark 2: Maximizing average SNR was discussed in [1] Similarly, when the original problem of maximizing weighted sum rate degenerates to maximizing weighted SNR, optimization objective is

$$
\sum_{i=1}^{N} \alpha_{i} \mathbf{w}^{\mathrm{H}} \mathbf{Q}_{i} \mathbf{w}=\mathbf{w}^{\mathrm{H}}\left(\sum_{i=1}^{N} \alpha_{i} \mathbf{Q}_{i}\right) \mathbf{w} \triangleq \mathbf{w}^{\mathrm{H}} \mathbf{Q} \mathbf{w}
$$

To achieve the maximum weighted SNR, the optimal beamformer is the normalized eigenvector corresponding to the maximum eigenvalue of $\mathbf{Q}$, which is a kind of simplification.

\section{TheORETICAL ANALysis}

\section{A. Properties of the optimal solution}

In (3), the domain of $f(\mathbf{w})$ is $\mathbb{C}^{M \times 1}$, while $f(\mathbf{w})$ is continuous and twice differentiable, whose Hessian is

$$
\nabla_{\mathbf{w}}^{2} f(\mathbf{w})=-\sum_{i=1}^{N} \frac{\alpha_{i}}{\left(1+\mathbf{w}^{\mathrm{H}} \mathbf{Q}_{i} \mathbf{w}\right)^{2}} \mathbf{Q}_{i}
$$

Because $\nabla_{\mathbf{w}}^{2} f(\mathbf{w})$ is negative semidefinite, objective function is concave. In addition, the feasible set is convex. Thus the original problem (3) is not convex [6].

Some properties of the optimal solution are derived.

Theorem 1. The optimal beamformer of maximizing weighted sum rate must lie in the subspace spanned by each user's channel vector.

Proof: Assume the optimal beamformer of maximizing weighted sum rate scheme does not lie within the subspace spanned by each user's channel vectors, which means $\mathbf{w}_{\text {opt }}$ can be expressed as $\mathbf{w}_{\text {opt }}=\sqrt{\gamma} \mathbf{w}_{1}+\sqrt{1-\gamma} \mathbf{w}_{2}, 0 \leq$ $\gamma<1$ where $\left\|\mathbf{w}_{\text {opt }}\right\|=\left\|\mathbf{w}_{1}\right\|=\left\|\mathbf{w}_{2}\right\|$, and $\mathbf{w}_{1} \in$ $\operatorname{span}\left(\mathbf{h}_{1}, \cdots, \mathbf{h}_{\mathrm{N}}\right), \mathbf{w}_{2} \in \operatorname{null}\left(\mathbf{h}_{1}, \cdots, \mathbf{h}_{\mathrm{N}}\right)$. As defined in (3),
It is knowable that $f\left(\mathbf{w}_{\text {opt }}\right)>f\left(\mathbf{w}_{1}\right)$, which implies $\mathbf{w}_{\text {opt }}$ is not optimal, contradicting the assumption.

Theorem 2. The optimal beamformer should satisfy $\|\mathrm{w}\|^{2}=$ 1 , which means the base station works with maximum power.

\section{B. Closed-form solutions under some usual special cases}

Introduce the Lagrange function $\mathcal{L}(\mathbf{w}, \lambda)$ of problem (3), where $\lambda$ is Lagrange multiplier. We obtain the Karush-KuhnTucker (KKT) conditions of (3)

$$
\left\{\begin{array}{l}
\|\mathbf{w}\|^{2} \leq 1, \lambda \geq 0, \lambda\left(\|\mathbf{w}\|^{2}-1\right)=0 \\
\nabla_{\mathbf{w}} \mathcal{L}(\mathbf{w}, \lambda)=0
\end{array}\right.
$$

where

$$
\nabla_{\mathbf{w}} \mathcal{L}(\mathbf{w}, \lambda)=\left[\lambda \mathbf{I}_{M}-\sum_{i=1}^{N} \frac{\alpha_{i}}{1+\mathbf{w}^{\mathrm{H}} \mathbf{Q}_{i} \mathbf{w}} \mathbf{Q}_{i}\right] \mathbf{w}=0
$$

It implies that $\mathbf{w}$ is the eigenvector of a linear combination of matrix $\mathbf{Q}_{1}, \cdots, \mathbf{Q}_{N}$. Since the original problem is nonconvex, some special cases are considered.

1) All the channels are poor: In this case, $\mathrm{w}^{\mathrm{H}} \mathbf{Q}_{i} \mathbf{w}$ is much less than 1. Thus (8) can be simplified as

$$
\nabla_{\mathbf{w}} \mathcal{L}(\mathbf{w}, \lambda) \approx\left[\lambda \mathbf{I}_{M}-\sum_{i=1}^{N} \alpha_{i} \mathbf{Q}_{i}\right] \mathbf{w}=0
$$

Hence $\mathbf{w}$ should be the normalized eigenvector corresponding to the maximum eigenvalue of matrix $\sum_{i=1}^{N} \alpha_{i} \mathbf{Q}_{i}$, which is equivalent to maximizing weighted SNR as (5).

2) Users on orthogonal directions with high SNR: In practical system, users feedback channels through codewords, which includes numerous orthogonal codewords, thus this is a usual case. Assume $\mathbf{v}_{1}, \cdots, \mathbf{v}_{K}$ is a set of normal orthogonal basis of $\operatorname{span}\left(\mathbf{h}_{1}, \cdots, \mathbf{h}_{N}\right)$. According to Theorem $1, \mathbf{w} \in \operatorname{span}\left(\mathbf{h}_{1}, \mathbf{h}_{2}, \cdots, \mathbf{h}_{N}\right)$, thus $\mathbf{w}$ can be expressed as

$$
\mathbf{w}=\sum_{k=1}^{K} a_{k} \mathbf{v}_{k}
$$

In this case, we divide them into $K$ groups. Assume there are $N_{k}$ users on $k$-th direction, whose normal orthogonal basis is $\mathbf{v}_{k}$. Denote original index $i$ by $(k, j)$, which means $j$-th user on $k$-th direction.

For $k=1, \cdots, K, j=1, \cdots, N_{k}$, we have

$$
\mathbf{h}_{(k, j)}=\left\|\mathbf{h}_{(k, j)}\right\| \mathrm{e}^{\mathrm{j} \gamma_{(k, j)}} \mathbf{v}_{k}
$$

Substituting (10) and (11) to (3), original problem becomes

$\begin{cases}\min _{\mathbf{w}} & -\sum_{k=1}^{K}\left[\alpha_{(k, j)} \sum_{j=1}^{N_{k}} \log _{2}\left(1+\frac{P\left\|\mathbf{h}_{(k, j)}\right\|^{2}}{\sigma_{(k, j)}^{2}}\left|a_{i}\right|^{2}\right)\right] \\ \text { s.t. } & \sum_{k=1}^{K}\left|a_{k}\right|^{2} \leq 1\end{cases}$

Denote $\mathbf{c}=\left(c_{1}, \cdots, c_{K}\right)^{\mathrm{T}}=\left(\left|a_{1}\right|^{2}, \cdots,\left|a_{K}\right|^{2}\right)^{\mathrm{T}}$, problem (12) can be converted to following equivalent form

$$
\begin{cases}\min _{\mathbf{c}} & -\sum_{k=1}^{K}\left[\alpha_{(k, j)} \sum_{j=1}^{N_{k}} \log _{2}\left(1+\frac{P\left\|\mathbf{h}_{(k, j)}\right\|^{2}}{\sigma_{(k, j)}^{2}} c_{k}\right)\right] \\ \text { s.t. } & \sum_{k=1}^{K} c_{k} \leq 1 \\ & c_{k} \geq 0, i=1, \cdots, K\end{cases}
$$


Thus the objective function is converted to a logarithmic linear function, which is a convex optimization problem. The Lagrange function of problem (13) is

$$
\begin{aligned}
\mathcal{L}(\mathbf{c}, \lambda, \boldsymbol{\mu})= & -\sum_{k=1}^{K}\left[\alpha_{(k, j)} \sum_{j=1}^{N_{k}} \log _{2}\left(1+\frac{P\left\|\mathbf{h}_{(k, j)}\right\|^{2}}{\sigma_{(k, j)}^{2}} c_{k}\right)\right] \\
& +\lambda\left(\mathbf{1}_{K}^{\mathrm{T}} \mathbf{c}-1\right)-\boldsymbol{\mu}^{\mathrm{T}} \mathbf{c}
\end{aligned}
$$

where $\lambda$ and $\boldsymbol{\mu}$ are Lagrange multipliers.

We obtain the KKT conditions of (13)

$$
\left\{\begin{array}{l}
\mathbf{1}_{K}^{\mathrm{T}} \mathbf{c} \leq 1, \lambda \geq 0, \lambda\left(\mathbf{1}_{K}^{\mathrm{T}} \mathbf{c}-1\right)=0 \\
c_{k} \geq 0, \mu_{k} \geq 0, \mu_{k} c_{k}=0, k=1, \cdots, K \\
\nabla_{c_{k}} \mathcal{L}(\mathbf{c}, \lambda, \boldsymbol{\mu})=0, k=1, \cdots, K
\end{array}\right.
$$

Thus we have

$$
\nabla_{c_{k}} \mathcal{L}(\mathbf{c}, \lambda, \boldsymbol{\mu})=-\sum_{j=1}^{N_{k}} \frac{\alpha_{(k, j)}}{\frac{\sigma_{(k, j)}^{2}}{P\left\|\mathbf{h}_{(k, j)}\right\|^{2}}+c_{k}}+\lambda-\mu_{k}=0
$$

By noting that $\mu_{k}$ acts as a slack variable, we get

$$
\left(\sum_{j=1}^{N_{k}} \frac{\alpha_{(k, j)}}{\frac{\sigma_{(k, j)}^{2}}{P\left\|\mathbf{h}_{(k, j)}\right\|^{2}}+c_{k}}-\lambda\right) c_{k}=0, k=1, \cdots, K
$$

With high SNR $\left(\frac{\sigma_{(k, j)}^{2}}{P\left\|\mathbf{h}_{(k, j)}\right\|^{2}}\right.$ is negligible) and power constraint Theorem 2, we get $c_{k} \approx \sum_{j=1}^{N_{k}} \alpha_{(k, j)}, k=1, \cdots, K$, which implies that $c_{k}$ is sum of the weights on $k$-th direction. Because $c_{k}=\left|a_{k}\right|^{2}$, we obtain

$$
\mathbf{w}_{\text {opt }}=\sum_{k=1}^{K}\left(\sqrt{\sum_{j=1}^{N_{k}} \alpha_{(k, j)}} \mathrm{e}^{\mathrm{j} \theta_{k}} \mathbf{v}_{k}\right)
$$

where $\theta_{1}, \cdots, \theta_{K}$ is any real number in $[0,2 \pi]$.

3) Channels are pairwise orthogonal with any SNR: In this case, $\mathbf{h}_{1} /\left\|\mathbf{h}_{1}\right\|, \cdots, \mathbf{h}_{N} /\left\|\mathbf{h}_{N}\right\|$ is a set of standard orthogonal basis of $\operatorname{span}\left(\mathbf{h}_{1}, \cdots, \mathbf{h}_{N}\right)$, and the dimension of $\operatorname{span}\left(\mathbf{h}_{1}, \cdots, \mathbf{h}_{N}\right)$ is $K=N$. Thus (10) can be expressed as

$$
\mathbf{w}=\sum_{k=1}^{K} a_{k} \frac{\mathbf{h}_{k}}{\left\|\mathbf{h}_{k}\right\|}
$$

Then (17) can be written as

$$
\left(\lambda-\frac{\alpha_{k}\left\|\mathbf{h}_{k}\right\|^{2}}{\sigma_{k}^{2} / P+c_{k}\left\|\mathbf{h}_{k}\right\|^{2}}\right) c_{k}=0, k=1, \cdots, K
$$

Following theorem holds:

Theorem 3. If channel vectors are pairwise orthogonal, then w satisfies $c_{k} \neq 0$ for $k=1, \cdots, K$.

Proof: For convenience and without loss of generality, we assume $\mathbf{w}_{\text {opt }}$ satisfies $\mathbf{h}_{1}^{\mathrm{H}} \mathbf{w}_{\text {opt }}=0$, hence $\mathbf{w}_{\text {opt }}$ can be expressed as $\mathbf{w}_{\mathrm{opt}}=\sum_{k=2}^{K} b_{k} \mathbf{h}_{k}$. Assume another beamforming vector $\mathbf{w}^{\prime}$ can be written as $\mathbf{w}^{\prime}=\left(\sqrt{\beta} \mathbf{w}_{\text {opt }} /\left\|\mathbf{w}_{\text {opt }}\right\|+\right.$ $\left.\sqrt{1-\beta} \mathbf{h}_{1} /\left\|\mathbf{h}_{1}\right\|\right)\left\|\mathbf{w}_{\text {opt }}\right\|$, where $0 \leq \beta \leq 1$. Then we discuss the value of $\beta$ when $\mathbf{w}^{\prime}$ is optimal. If $\beta$ can never be 1 , the assumption is false. Regard $f\left(\mathbf{w}^{\prime}\right)$ as a function of variable $\beta$, which is continuous and differentiable when $0 \leq \beta \leq 1$. In addition, $\frac{\mathrm{d}}{\mathrm{d} \beta} f\left(\mathbf{w}^{\prime}\right)$ increases continuously as $\beta$ increases, and $\left.\frac{\mathrm{d}}{\mathrm{d} \beta} f\left(\mathbf{w}^{\prime}\right)\right|_{\beta=0}<0,\left.\frac{\mathrm{d}}{\mathrm{d} \beta} f\left(\mathbf{w}^{\prime}\right)\right|_{\beta=1}>0$, i.e., the derivative changes from negative to positive. It implies that the function value first descends then increases, minimum point is in the interval $(0,1)$. Thus $\beta=1$ can never be satisfied.

According to Theorem 3, (20) can be simplified as

$$
\lambda-\frac{\alpha_{k}\left\|\mathbf{h}_{k}\right\|^{2}}{\sigma_{k}^{2} / P+c_{k}\left\|\mathbf{h}_{k}\right\|^{2}}=0, k=1, \cdots, K
$$

Considering the power constraint and $c_{k} \geq 0$, we have $c_{k}=$ $\alpha_{k}$ for $k=1, \cdots, K$ and

$$
\mathbf{w}_{\mathrm{opt}}=\sum_{k=1}^{K}\left(\sqrt{\alpha_{k}} \mathrm{e}^{\mathrm{j} \theta_{k}} \frac{\mathbf{h}_{k}}{\left\|\mathbf{h}_{k}\right\|}\right)
$$

where $\theta_{1}, \cdots, \theta_{K}$ is any real number in $[0,2 \pi]$.

\section{ITERATIVE ALGORITHM}

For general case of problem (3), we propose following iterative algorithm based on modified gradient descent method:

1) Initialization: iteration counter is $k=1$, initial beamformer $\mathbf{w}_{1}$ is set as the optimal solution of maximizing weighted SNR, i.e., the normalized eigenvector corresponding to the maximum eigenvalue of $\sum_{i=1}^{N} \alpha_{i} \mathbf{Q}_{i}$ in (5);

2) Determine descent direction: compute the gradient of $f\left(\mathbf{w}_{k}\right)$ at $\mathbf{w}_{k}$. Set the descent direction of $k$-th iteration as the negative gradient, i.e.

$$
\Delta \mathbf{w}_{k}=-\nabla f\left(\mathbf{w}_{k}\right)=\sum_{i=1}^{N}\left(\alpha_{i} \frac{\mathbf{Q}_{i} \mathbf{w}_{k}}{1+\mathbf{w}_{k}^{\mathrm{H}} \mathbf{Q}_{i} \mathbf{w}_{k}}\right)
$$

3) Determine the step size $\eta_{k}$ of $k$-th iteration by backtracking line search in [6]: initialize $\eta_{k}$ with $\eta_{k}=1$, if $f\left(\mathbf{w}_{k}+\eta_{k} \Delta \mathbf{w}_{k}\right)>f\left(\mathbf{w}_{k}\right)+\rho \eta_{k} \nabla f\left(\mathbf{w}_{k}\right)^{\mathrm{T}} \Delta \mathbf{w}_{k}$, update $\eta_{k}$ as $\eta_{k}:=\xi \eta_{k}$ until above inequality is violated, where $\rho \in(0,0.5)$ and $\xi \in(0,1)$ are constant values, then regard $\eta_{k}$ as the step size.

4) Update: $\mathbf{w}_{k+1}=\mathbf{w}_{k}+\eta_{k} \Delta \mathbf{w}_{k}$, and normalize it, i.e., $\mathbf{w}_{k+1}:=\mathbf{w}_{k+1} /\left\|\mathbf{w}_{k+1}\right\|$

5) Judgement: If the terminal condition

$$
\left|\frac{f\left(\mathbf{w}_{k+1}\right)-f\left(\mathbf{w}_{k}\right)}{f\left(\mathbf{w}_{k}\right)}\right|<\varepsilon
$$

is fulfilled, then the iteration stops, where $\varepsilon$ is a small positive constant value; otherwise the algorithm switches to step 2 to continue iteration and $k:=k+1$.

\section{Simulation Results}

In this section, the performance of the proposed iterative algorithm is compared with previous schemes: open-loop transmission (i.e., w is $\frac{1}{\sqrt{M}} \mathbf{1}_{M}$ ), SDR beamforming [2], successive beamforming [3] and maximizing average SNR 
scheme [1], with simulation parameters as follows: 4 antennas at transmitter, $P / \sigma_{i}^{2}$ for all $i=1, \cdots, N$ is set to be $10 \mathrm{~dB}$.

Minimum rate with respect to the number of users is shown in Fig. 1. The proposed scheme in this paper shows excellent performance compared to other schemes. That is, although the proposed scheme does not guarantee the worst user, the selection of weight tends to focus on the worse users.

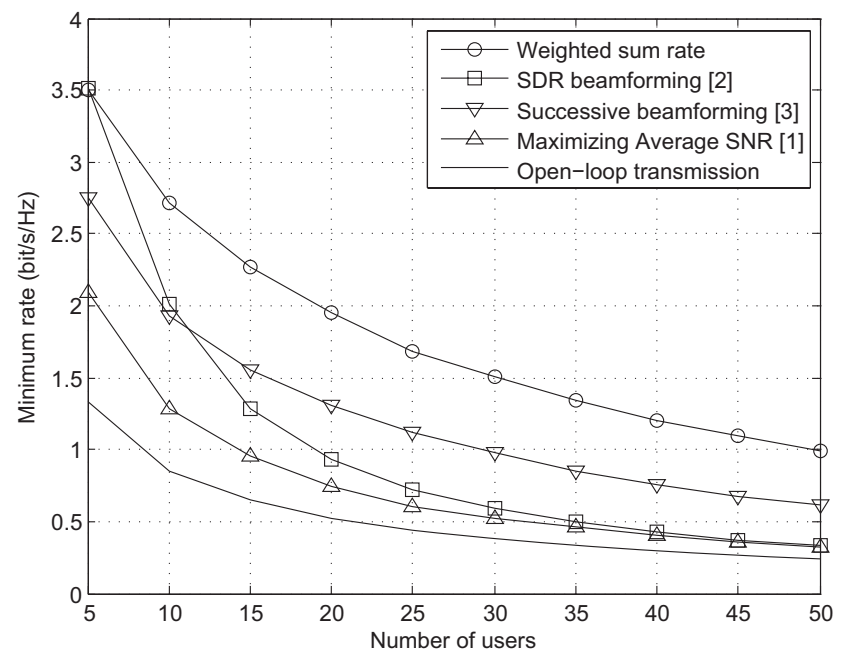

Fig. 1. Minimum rate versus the number of users

Fig. 2 shows the average rate of different schemes. The proposed scheme yields best average rate among all the schemes.

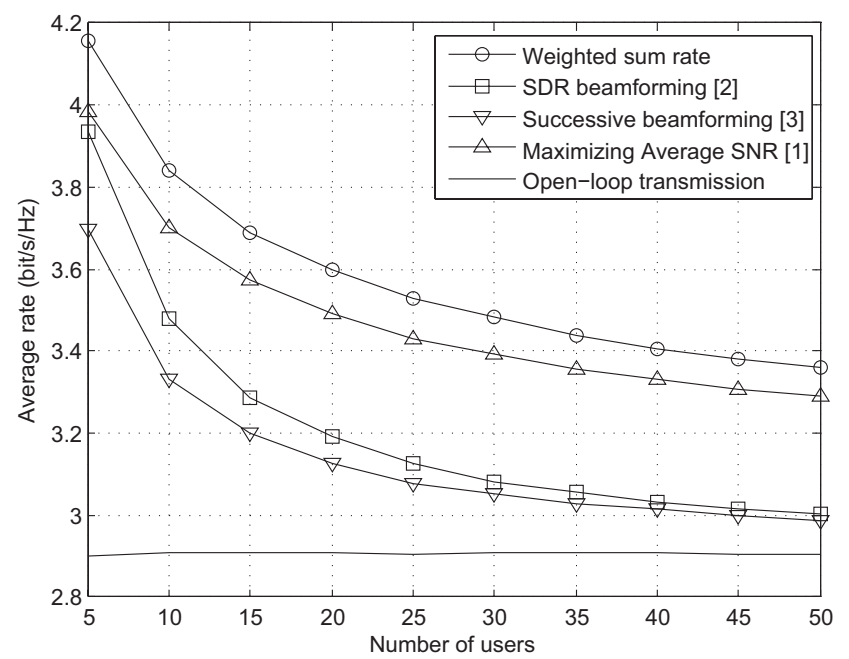

Fig. 2. Average rate versus the number of users

The cumulative distribution functions of rate with 10 users are shown in Fig. 3. The proposed scheme improves the overall rate remarkably compared to previous schemes except the maximizing average SNR scheme. Although the maximizing average SNR scheme overcomes the proposed scheme with high rate, users with poor channels may be neglected, leading to many users with extremely low rate. It implies that, the proposed scheme can guarantee the overall performance as well as the fairness.

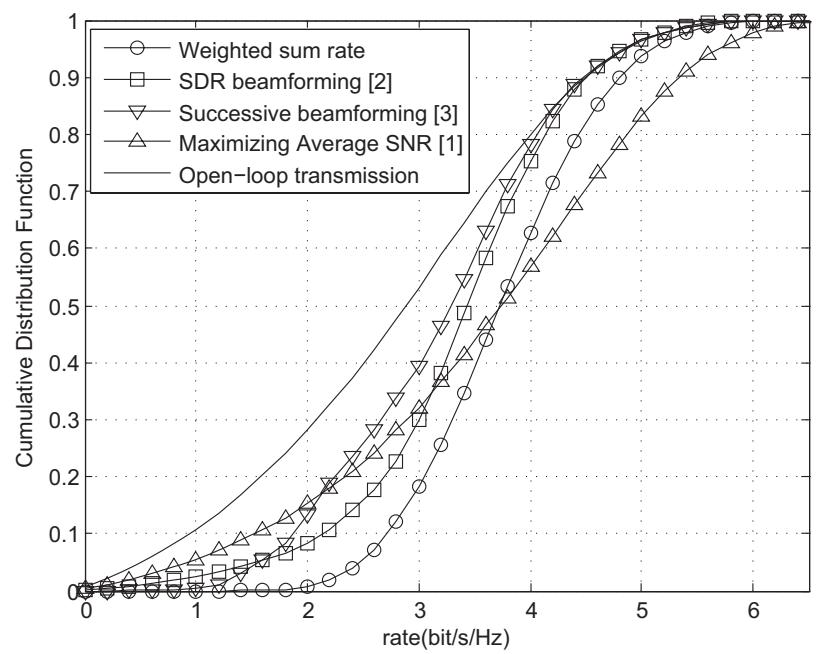

Fig. 3. Cumulative distribution functions of rate with 10 users

Fig. 4 shows the fairness, which is defined by fairness $=$ $\left(\sum_{i=1}^{N} R_{i}\right)^{2} /\left(N \sum_{i=1}^{N} R_{i}^{2}\right)$ proposed in [7]. The proposed scheme is fairer than others, especially with a large number of users.

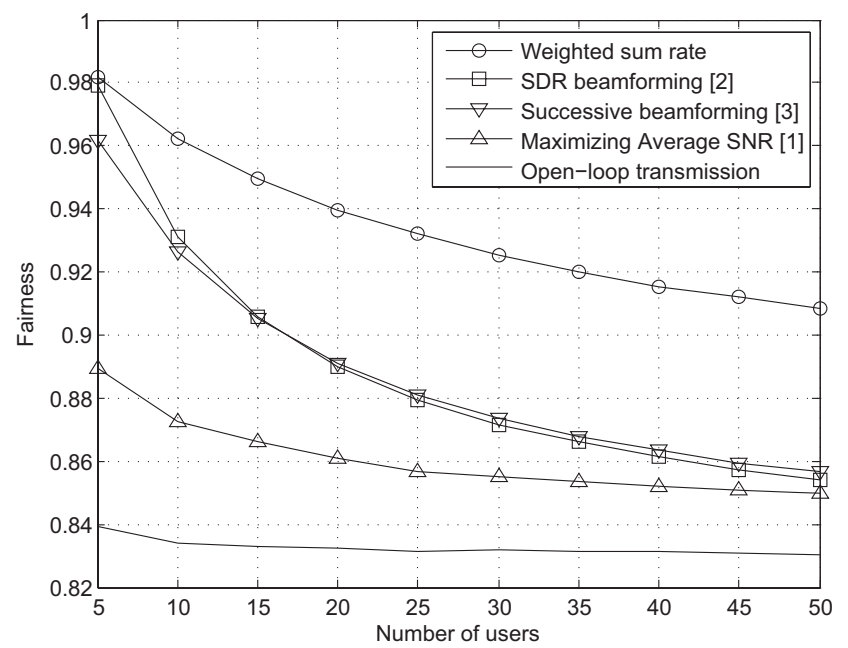

Fig. 4. Fairness versus the number of users

\section{CONCLUSION}

In this paper, maximizing weighted sum rate is proposed for beamformer design in single group multicast systems. It can provide performance gain compared with previous schemes because all the user are considered with different priority. An intuitional and satisfactory weight is chosen in this paper. Future work can focus on finding more suitable weights, and the theoretical relation between maximizing weighted sum rate and maximizing minimum rate should be discussed deeper. 


\section{ACKNOWLEDGMENT}

This work has been supported by Key Special Project of National Science and Technology (No. 2010ZX03003-002-01, 2010ZX03003-004-02), National Nature Science Foundation of China (No.61172077 \& No. 60972023), and Research Fund of National Mobile Communications Research Laboratory, Southeast University (No. 2012A06).

\section{REFERENCES}

[1] M. J. Lopez, "Multiplexing, scheduling, and multicasting strategies for antenna arrays in wireless networks," Ph.D. dissertation, Dept. Elect. Eng. and Comp. Sci., Mass. Inst. Technol. (MIT), Cambridge, 2002.

[2] N. D. Sidiropoulos, T. N. Davidson, and L. Zhi-Quan, "Transmit beamforming for physical-layer multicasting," IEEE Trans. Signal Processing, vol. 54, pp. 2239-2251, 2006.

[3] K. Il Han, D. J. Love, and P. Seung Young, "Optimal and Successive Approaches to Signal Design for Multiple Antenna Physical Layer Multicasting," IEEE Trans. Commun., vol. 59, pp. 2316-2327, 2011.

[4] E. Karipidis, N. D. Sidiropoulos, and Z. Q. Luo, "Far-field multicas beamforming for uniform linear antenna arrays," IEEE Trans. Signal Processing, vol. 55, pp. 4916-4927, 2007.

[5] N. Jindal and Z. Q. Luo, "Capacity limits of multiple antenna multicast," IEEE International Symposium on Information Theory, pp. 1841-1845, 2006.

[6] S. P. Boyd and L. Vandenberghe, Convex optimization. Cambridge, U. K. Combridge Univ. Press, 2004.

[7] R. Jain, D. M. Chiu, and W. Hawe, "A quantitative measure of fairnes and discrimination for resource allocation in shared computer systems," DEC Research Report TR-301, 1984. 